\title{
O Potencial da Gamificação para Aumentar a Competitividade dos Destinos Turísticos: revisão de literatura baseada na Scopus
}

\author{
Viviane da Silva Souzaa \\ Celeste Maria Dias Amorim Varumb \\ Celeste Eusébio ${ }^{c}$
}

\begin{abstract}
Resumo
A gamificação surgiu com o propósito de gerar envolvimento, lealdade e, acima de tudo, proporcionar uma experiência marcante para turistas e visitantes. Em vista disso, os destinos turísticos tornaram-se mais sensíveis às oportunidades de criação de novos serviços e produtos gamificados na indústria do turismo. Nesse cenário, este artigo objetiva analisar os estudos científicos (teóricos e empíricos) sobre a gamificação aplicada ao turismo com a finalidade de identificar elementos que justifiquem o potencial da gamificação para gerar competitividade nos destinos turísticos. Para atingir o objetivo, executaram-se pesquisas eletrônicas na base de dados especializada Scopus, atingindo-se, ao término da pesquisa, um agregado de onze documentos científicos identificados como significativos para a abordagem deste estudo. Deste modo, esta revisão exploratória da literatura trouxe as seguintes conclusões: (1) identificação de tendências na literatura sobre gamificação no turismo; (2) autores e publicações que mais se destacam nessa área; (3) objetivos, contextos e abordagens da gamificação no setor; (4) o potencial da gamificação para a competitividade; e, por fim, (5) a indicação das abordagens metodológicas adotadas nos estudos analisados. Além disso, permitiuse realizar uma síntese do conhecimento sobre a temática, o que possibilitou a percepção do que já se sabe sobre o tema e o que se precisa saber, além de apontar direções para investigações futuras e algumas limitações de investigações nessa área.
\end{abstract}

Palavras-chave: Gamificação; Destinos turísticos; Competitividade.

\begin{abstract}
The potential of gamification to increase the competitiveness of tourist destinations: literature review based on Scopus

Gamification has emerged with the purpose of generating involvement, loyalty and, above all, providing a remarkable experience for tourists and visitors. In view of this, tourist destinations have become more sensitive to the opportunities to create new services and
\end{abstract}

a. Doutoranda em Turismo pela Universidade de Aveiro. Mestre em Administração pela Universidade Federal Rural de Pernambuco (UFRPE). Bacharel em Turismo pela Universidade Federal de Pernambuco (UFPE). Aveiro, Portugal. E-mail: vivianne.turismologa@gmail.com

b. Doutora em Economia. Mestre em International Business and Economic Integration pela University of Reading (UK). Graduada em Economia pela Universidade de Évora. Professora de Economia na Universidade de Aveiro. Aveiro, Portugal. E-mail: camorim@ua.pt

c. Doutora em Turismo pela Universidade de Aveiro. Professora Auxiliar na Universidade de Aveiro e Investigadora da Unidade de Investigação GOVCOPP (UA). Aveiro, Portugal. E-mail: celeste. eusebio@ua.pt 
gamified products in the tourism industry. In this scenario, this article aims to analyze the scientific studies (theoretical and empirical) on gamification applied to tourism in order to identify elements that justify the potential of gamification to generate competitiveness in tourist destinations. In order to achieve this goal, an electronic research was carried out in the specialized Scopus database, reaching, at the end of the research, an aggregate of eleven scientific documents identified as significant for the approach of this study. Thus, this exploratory review of the literature brought the following conclusions: (1) identification of trends in the literature on tourism gamification; (2) authors and publications that stand out most in this area; (3) goals, contexts, and approaches to gamification in the industry; (4) the potential of gamification for competitiveness; and, finally, (5) the indication of the methodological approaches adopted in the studies analyzed. In addition, it was possible to make a synthesis of the knowledge on the subject, which made possible the perception of what is already known about the subject and what one needs to know, besides pointing out directions for future investigations and some limitations of investigations in this area.

Keywords: Gamification; Tourist destinations; Competitiveness.

\section{Resumen}

\section{El potencial de gamificación para aumentar la competitividad de destinos turísticos: revisión de la literatura basada en Scopus}

La gamificación se acercó con el propósito de generar participación, la lealtad y sobre todo proporcionar una experiencia notable para los turistas y visitantes. La vista de esto, los destinos turísticos se han vuelto más sensibles a las oportunidades para crear nuevos servicios y productos en la industria gamificados turismo. En este escenario, este artículo tiene como objetivo analizar los estudios científicos (teóricos y empíricos sobre gamificación) aplicado en el turismo con el fin de identificar elementos de apoyo al potencial de gamificación para generar competitividad en los destinos turísticos. Para lograr el objetivo, que se llevaron a cabo investigaciones en una base de datos electrónica especializada, Scopus, de llegar al final de la investigación un total de once trabajos científicos indicados como significativos para el enfoque de este estudio. Por lo tanto, esta revisión sistemática de la literatura permitió a las siguientes conclusiones: (1) la identificación de las tendencias en la literatura sobre gamificación en el turismo; (2) autores y publicaciones que se destacan en este ámbito; (3) los objetivos, contextos y enfoques de gamificación en el sector; (4) el potencial de gamificación para la competitividad y, por último, (5) apunta hacia arriba los enfoques metodológicos adoptados en los estudios analizados. También permite realizar una síntesis de los conocimientos sobre el tema, dando la oportunidad de conocer lo que ya se sabe sobre el tema y lo que necesita saber, y señalar direcciones para futuras investigaciones y algunas limitaciones de la investigación en esta área.

Palabras clave: Gamificación; Destinos turísticos; Competitividad.

\section{INTRODUÇÃO}

Este artigo converge-se com as recentes investigações sobre as novas tecnologias e marketing, nomeadamente no que concerne ao potencial da gamificação como ferramenta para aumentar a competitividade dos destinos turísticos. Um novo modelo de turismo concentra-se na experiência experimentada pelos turistas em seus destinos, e os exemplos mais bem-sucedidos são os serviços e produtos que utilizam a tecnologia para proporcionar experiências eternas e únicas (KOVACEVIC et al., 2014). Em busca de atender a essas mudanças de atitudes, comportamentos e exigências dos turistas, a oferta do setor do turismo necessita 
se posicionar diante desse novo mercado. Isto é, há a necessidade de entender esses novos comportamentos, hábitos, preferências, desejos e necessidades para que consigam delinear novas estratégias que ajudem a oferta a permanecer atualizada e inovadora diante da atual realidade.

É nesse cenário que os jogos têm sido utilizados na indústria do turismo, para proporcionar uma experiência marcante aos turistas. 0 novo elemento que altera o paradigma do marketing e dos negócios chama-se Gamification, termo em inglês que possui como definição mais difundida: uso de elementos e design do jogo em contextos não-jogo (DETERDING et al., 2011a). Propositalmente, a gamificação foi elaborada para atingir efeitos motivacionais ou de comportamento para atrair mais clientes, aumentar sua experiência e estimular a retenção de consumidores no setor empresarial (ASQUER; KRACHKOVSKAYA, 2015). Por exemplo, o serviço baseado em localização Foursquare, utiliza elementos de design do jogo em contextos não-jogo para motivar, aumentar a atividade do usuário e gerar envolvimento (DETERDING et al., 2011a). Diante do reconhecimento sobre a importância e o impacto que a gamificação vem desempenhando no contexto da gestão, saúde, educação e turismo (XU et al., 2015), neste último caso, percebe-se o início de uma estreita relação com a indústria de jogos; uma vez que no setor é crescente o número de serviços e produtos gamificados em desenvolvimento e outros já disponíveis.

Diante disso, este artigo destina-se aos seguintes objetivos: (1) identificar tendências na literatura sobre gamificação no turismo; (2) apontar autores e publicações que mais se destacam nessa área; (3) detectar objetivos, contextos e abordagens da gamificação no setor do turismo; (4) identificar o potencial da gamificação para a competitividade e, por fim, (5) apontar as abordagens metodológicas adotadas nos estudos analisados. Para atingir tais finalidades, fez-se necessária uma revisão sistemática de literatura na base de dados Scopus ${ }^{1}$.

Justifica-se a realização deste estudo pelos seguintes motivos: no que compete às pesquisas acadêmicas sobre gamificação, Xu et al. (2015) explicam que ainda são muito limitadas, em especial no setor de turismo. No que concerne à construção do conhecimento para o marketing e turismo enquanto ciência, o estudo realizado para este artigo parece plausível, na medida em que está em consonância com as prioridades de pesquisa estabelecidas pelos principais centros de estudos como Massachusetts Institute of Technology (MIT), Cornell University, Tampere Institut of Technology (TUT), entre outros. Por fim, tratando-se do interesse acadêmico sobre o tema, este artigo contribui com as áreas de conhecimento do turismo e do marketing.

O artigo está estruturado da seguinte maneira: a segunda seção relata a metodologia aplicada neste estudo; a terceira seção expõe as seguintes subseções: (1) as tendências na literatura sobre gamificação no turismo, (2) noções sobre gamificação, (3) autores e publicações que mais se destacam, (4) objetivos, contextos e abordagens da gamificação no setor do turismo, (5) potencial da gamificação para a competitividade no turismo, (6) abordagens metodológicas adotadas nos estudos analisados; a quarta seção apresenta as principais conclusões, implicações para futuras pesquisas e limitações sobre o uso da gamificação para a competitividade nos destinos turísticos.

1. Base de dados de resumos, artigos e citações para jornais/revistas acadêmicos (https://www. scopus.com). 


\section{METODOLOGIA}

Nesta seção são indicados os caminhos percorridos para a operacionalização da pesquisa, especificando os meios pelos quais serão alcançados os dados para este estudo.

\section{Critérios para seleção dos documentos}

Com intenção de atingir os objetivos, fez-se necessário estabelecer critérios para a seleção dos documentos (Quadros 1 e 2). Efetuou-se uma busca na base de publicações científicas Scopus. As pesquisas foram feitas ao longo de um dia no mês de março de 2016, aplicando a palavra-chave "gamification" para a primeira etapa. Na segunda etapa, utilizaram-se os termos: "gamification and tourism", "gamification and tourism and competitiveness", "gamification and tourism and leisure", gamification and tourism or co-creation". Neste estudo, priorizou-se a base de dados com maior número de documentos, nesse caso, a Scopus; uma vez que poucos artigos sobre o tema foram identificados em outras bases, como, por exemplo, a Web of Science.

Quadro 1 - Critérios para seleção dos documentos: etapa um

\begin{tabular}{|l|l|}
\hline Temas & Etapas \\
\hline Base de publicações científicas & Scopus \\
\hline Palavras-chave & Gamification \\
\hline Idiomas & Inglês \\
\hline Horizonte temporal & $1960-2016$ \\
& $\begin{array}{l}\text { Computer Science; Social Sciences, Engineering, Mathematics, } \\
\text { Business, Management and Accounting, Medicine, Economics, } \\
\text { Econometrics and Finance, Psychology, Arts and Humanities, } \\
\text { Áreas }\end{array}$ \\
& $\begin{array}{l}\text { Science, Energy, Earth and Planetary Sciences, Nursing, } \\
\text { Multidisciplinary, Biochemistry, Genetics and Molecular } \\
\text { Biology, Materials Science, Agricultural and Biological } \\
\text { Sciences, Chemical Engineering, Neuroscience, Pharmacology, } \\
\text { Toxicology and Pharmaceutics, Physics and Astronomy, } \\
\text { Immunology and Microbiology, Undefined. }\end{array}$ \\
\hline Total de estudos & 1.496 documentos \\
\hline Fonte - Elaborado pelas autoras
\end{tabular}

Quadro 2 - Critérios para seleção dos documentos: etapa dois

\begin{tabular}{|l|l|}
\hline Temas & Etapas \\
\hline Base de publicações científicas & Scopus \\
\hline Palavras-chave & $\begin{array}{l}\text { "gamification and tourism", "gamification and tourism and } \\
\text { competitiveness", "gamification and tourism and leisure", } \\
\text { "gamification and tourism or co-creation". }\end{array}$ \\
\hline
\end{tabular}


Quadro 2 - Continuação

\begin{tabular}{|l|l|}
\hline Temas & Etapas \\
\hline Idiomas & Inglês \\
\hline Horizonte temporal & $1960-2016$ \\
\hline \multirow{2}{*}{ Áreas } & Computer Science, Business, Management and Accounting, \\
& Economics, Econometrics and Finance, Social Sciences, \\
& Arts and Humanities, Energy, Environmental Science, \\
Total de estudos & Mathematics. \\
\hline & 35 documentos \\
& Fonte - Elaborado pelas autoras
\end{tabular}

A fim de obter um resultado mais completo, os conceitos-chave foram pesquisados nos seguintes campos: título, resumo e palavras-chave dos artigos, nas áreas "Social Sciences \& Humanities", "Life Sciences", "Health Sciences" e "Physical Sciences". Por se tratar de uma temática recente na literatura e pouco explorada no âmbito do turismo, optou-se por não restringir os resultados, utilizando-se a opção: todo tipo de documento "Articles" ou "Articles in press", "Journals", "Book or Book chapter", "Article or conference paper", "Conference Review", "Editorial”, "Business Article", "Short Survey" e "Erratum". Porém, surgiu como fonte apenas "Conference Paper", "Article" e "Book Chapter".

Quanto ao idioma, em ambas as etapas seguiram-se os termos em inglês. Nas duas fases, não houve delimitação quanto ao horizonte temporal, assim, foram escolhidos todos os estudos publicados entre 1960 e 2016. Na primeira etapa foram encontrados 1.496 documentos e na segunda foram localizados 35. Depois dos resultados atingidos, seguiu-se uma terceira etapa, na qual foram lidos os resumos dos documentos e, tendo-os como base, foram incluídos apenas os temas que abordaram Tourism, leisure e co-creation.

Figura 1 - Etapas e quantidade de documentos

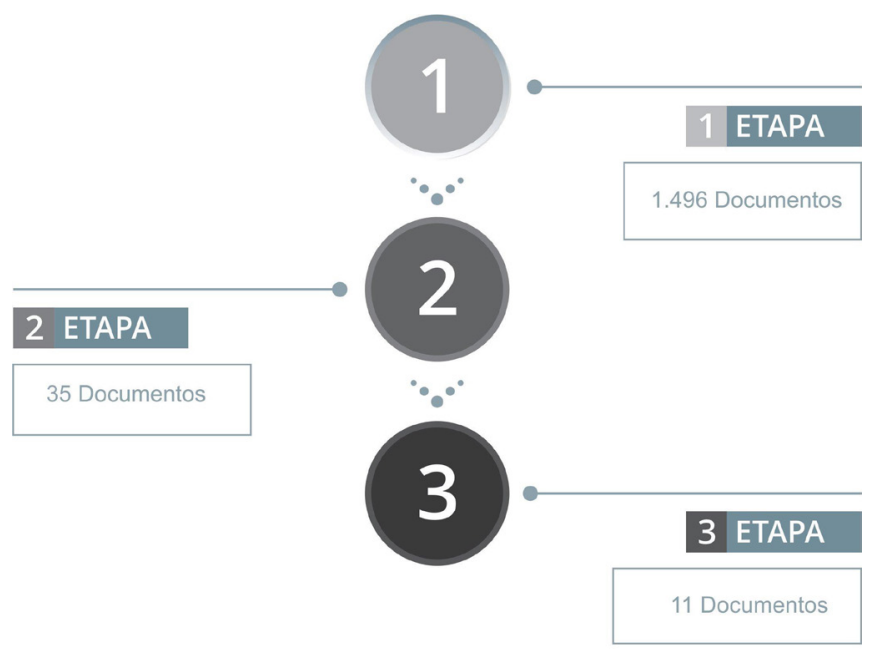

Fonte - Elaborado pelas autoras

Desse procedimento, resultaram-se onze documentos (Figura 1), que posteriormente foram lidos na íntegra (Quadro 3). Desses, há artigos de conferências 
(5), artigos (4) e capítulos de livro (2). É visível o domínio de artigo em conferência, pois se trata de um tema novo e ainda em exploração. Esses estudos foram publicados em conferências, livros e revistas científicas (Quadro 4).

\section{Quadro 3 - Autor x tema}

\begin{tabular}{|c|c|}
\hline Autor & Tema \\
\hline (XU et al., 2015) & $\begin{array}{l}\text { Tourists as Mobile Gamers: Gamification for } \\
\text { Tourism Marketing }\end{array}$ \\
\hline (SIGALA, 2015b) & $\begin{array}{l}\text { The application and program gamification } \\
\text { funware on trip planning and experiences: the } \\
\text { case of TripAdvisor's funware }\end{array}$ \\
\hline (NEGRUŞA et al., 2015) & $\begin{array}{l}\text { Exploring gamification techniques and } \\
\text { applications for sustainable tourism }\end{array}$ \\
\hline (SIGALA, 2015a) & $\begin{array}{l}\text { Gamification for crowdsourcing marketing } \\
\text { practices: Applications and benefits in tourism }\end{array}$ \\
\hline (KACHNIEWSKA, 2015) & $\begin{array}{l}\text { Gamification and social media as tools for tourism } \\
\text { promotion }\end{array}$ \\
\hline (KIRÁL’OVÁ, 2015) & $\begin{array}{l}\text { The place of gamification in tourism destinations' } \\
\text { marketing }\end{array}$ \\
\hline (BÁRCENA; SANFILIPPO, 2015) & $\begin{array}{l}\text { The audiovisual knowledge pill as a gamification } \\
\text { strategy in second language online courses }\end{array}$ \\
\hline (YAMAMOTO; YOSHIN; SONEHARA, 2015) & $\begin{array}{l}\text { Gamification effect of collection system for digital } \\
\text { photographs with geographic information which } \\
\text { utilizes land acquisition game }\end{array}$ \\
\hline (DÍAZ et al., 2014) & $\begin{array}{l}\text { The mystery of Elin. Incorporating a city cultural } \\
\text { program in history and heritage into a pervasive } \\
\text { game }\end{array}$ \\
\hline (GORDILLO et al., 2013) & The city as a learning gamified platform \\
\hline (GIOVANNELLA et al., 2013) & $\begin{array}{l}\text { Villard-de-lans: A case study for participatory } \\
\text { people-centered smart city learning design }\end{array}$ \\
\hline
\end{tabular}

Fonte - Elaborado pelas autoras

Quadro 4 - Revistas, livros e conferências

\begin{tabular}{l|l|l} 
Conferências & Artigos & Capítulo de livro \\
\hline $\begin{array}{l}\text { Proceedings Frontiers in } \\
\text { Education Conference Fie, }\end{array}$ & \\
$\begin{array}{l}\text { ACM International Conference } \\
\text { Proceeding Series, Idimt 2015 }\end{array}$ & Journal of Travel and Tourism, \\
$\begin{array}{l}\text { Information Technology and Society } \\
\text { Interaction and Interdependence }\end{array}$ & $\begin{array}{l}\text { Círculo de Linguística Aplicada a } \\
\text { la Comunicación, Sustainability } \\
\text { 23rd Interdisciplinary Information }\end{array}$ & $\begin{array}{l}\text { Handbook of } \\
\text { Research on }\end{array}$ \\
$\begin{array}{l}\text { Management Talks, Proceedings 2013 } \\
\text { IEEE 13th International Conference Advertising } \\
\text { on Advanced Learning Technologies } \\
\text { Icalt 2013 }\end{array}$ & Human-Computer Interaction & $\begin{array}{l}\text { Strategies in the } \\
\text { Social Media Age }\end{array}$ \\
\end{tabular}

Fonte - Elaborado pelas autoras 


\section{TENDÊNCIAS NA LITERATURA SOBRE GAMIFICAÇÃO NO TURISMO}

\section{Noções sobre gamificação}

Na base de dados do Scopus foi encontrado o total de 1.496 estudos sobre gamificação, conforme mencionado anteriormente (Quadro 1), destes, os primeiros emergiram entre 2011 e 2016 (Figura 2). Em 2012, na mesma base de dados, começaram a surgir estudos sobre a gamificação aplicada ao setor do turismo.

Figura 2 - Ano de publicação x números de documentos

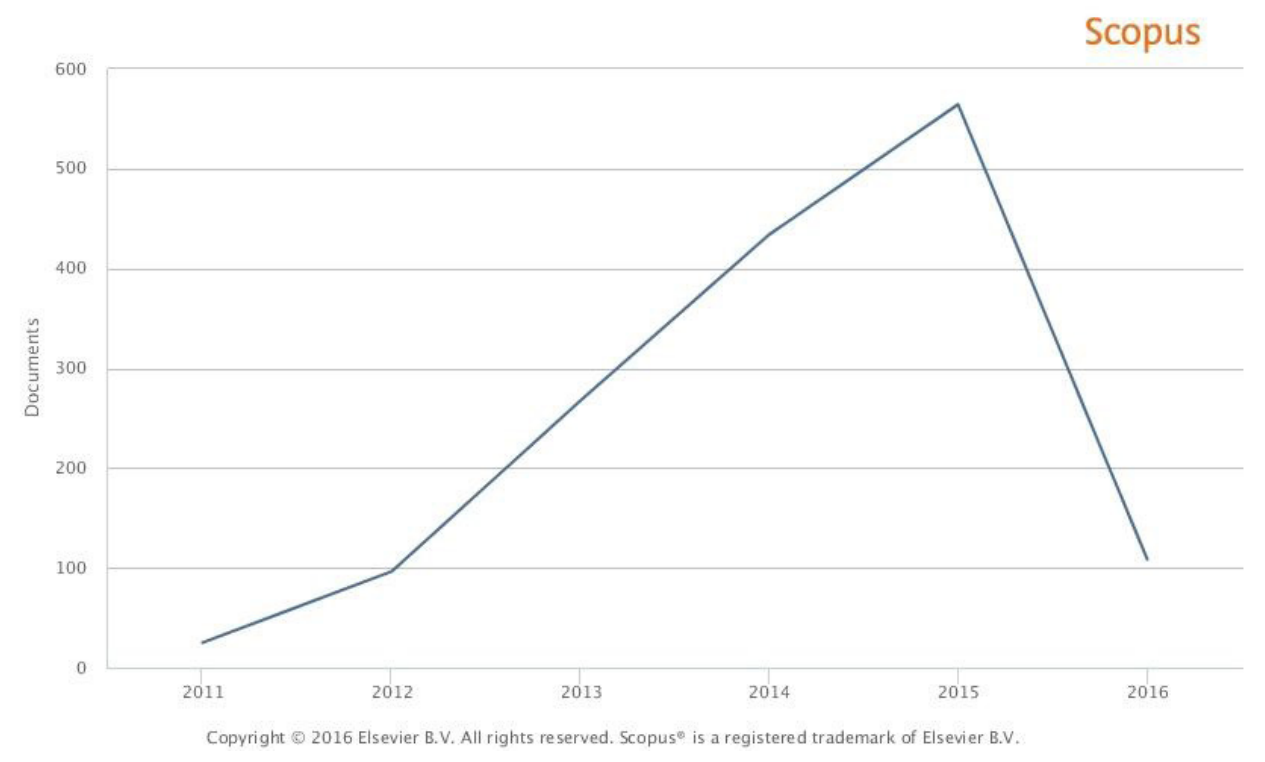

Fonte - Scopus (2016)

Verificou-se que o tema da gamificação despertou interesse de vários estudiosos, que conceituaram o assunto a partir de 2011. Pela revisão dos estudos identificados, complementada com a análise do trabalho de Ferreira (2015), pode-se apontar que existem, na literatura, diferentes definições de gamificação. A Figura 3 sintetiza as perspectivas mais dominantes.

Figura 3 - Evolução do conceito de gamificação

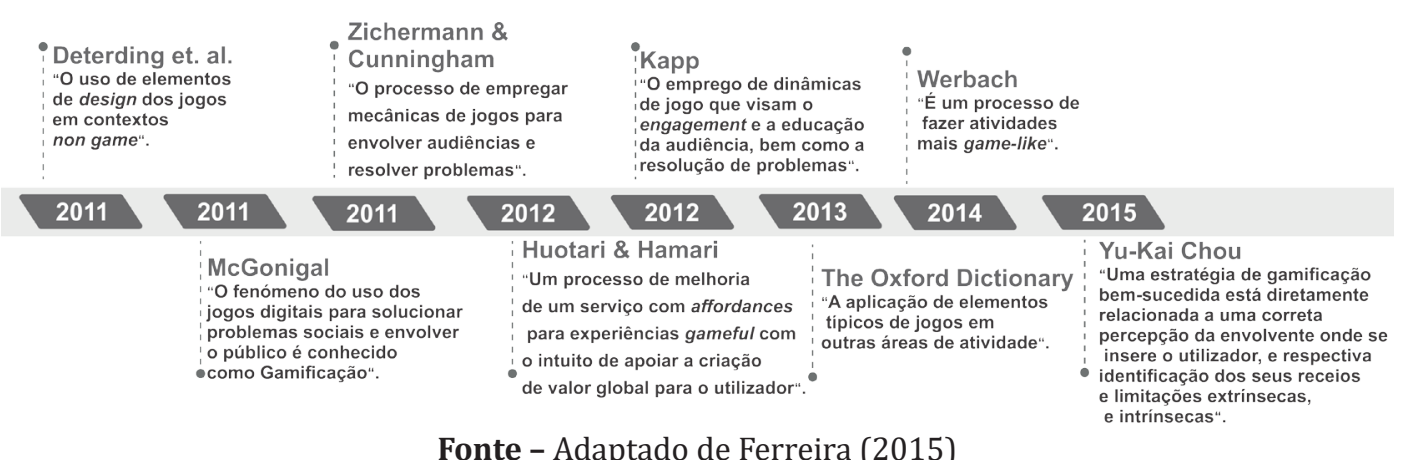

Fonte - Adaptado de Ferreira (2015) 
Apesar da falta de consenso no que diz respeito à definição da gamificação, o conceito mais difundido é o dado por Deterding et al., (2011b), que a define como o "uso de elementos de design do jogo em contextos fora do jogo", sendo estes os primeiros autores a conceituar o termo. Um ano após o surgimento dessa definição, Hamari e Huotari (2012) refinaram o conceito anterior para "um processo de reforço de um serviço com affordances ${ }^{2}$ para experiências divertidas, a fim de apoiar a criação de valor global dos usuários". A versão mais recente de Chou (2015) explica que, para uma estratégia de gamificação de sucesso, é necessário haver uma percepção correta da envolvente onde se insere o utilizador, isto é, faz-se indispensável entender o público-alvo ao que se pretende aplicar a gamificação e, além disso, identificar as motivações externas e internas dos utilizadores, visando melhor aplicabilidade. A evolução do conceito é fundamental para a compreensão e aplicabilidade mais abrangente sobre o tema.

Nos onze artigos identificados, constata-se que na literatura sobre gamificação em turismo, seguem as seguintes abordagens (Quadro 5).

Quadro 5 - Definições sobre gamificação utilizadas em artigos aplicados no turismo

\begin{tabular}{|c|c|}
\hline Autores & Definições \\
\hline (XU et al., 2015) & $\begin{array}{l}\text { "uso de elementos de design do jogo em contextos não-jogo" } \\
\text { (DETERDING et al., 2011a). }\end{array}$ \\
\hline (SIGALA, 2015b) & $\begin{array}{l}\text { "uso de elementos de design do jogo em contextos não-jogo" } \\
\text { (DETERDING et al., 2011a). } \\
\text { "um processo de reforço de um serviço com affordances para } \\
\text { experiências divertidas, a fim de apoiar a criação de valor global } \\
\text { dos usuários" (HAMARI; HUOTARI, 2012). }\end{array}$ \\
\hline (NEGRUŞA et al., 2015) & $\begin{array}{l}\text { "implementação e utilização de elementos de jogo (design e } \\
\text { técnicas), pensar o jogo, mecânica e análises do jogo, tecnologia } \\
\text { do jogo de computador em modelos de negócios, atividades } \\
\text { de enquadramento, processos, procedimentos, serviços e } \\
\text { outros, visando melhorar habilidade de usuário, a experiência, } \\
\text { o engajamento, eficácia e produtividade, além de puro } \\
\text { entretenimento tanto para empregado quanto para cliente" } \\
\text { (USKOV; SEKAR, 2014). } \\
\text { "uso de elementos de design do jogo em contextos fora do jogo" } \\
\text { (DETERDING et al., 2011b). }\end{array}$ \\
\hline (SIGALA, 2015a) & $\begin{array}{l}\text { "uso de elementos de design do jogo em contextos não-jogo" } \\
\text { (DETERDING et al., 2011a). }\end{array}$ \\
\hline (KACHNIEWSKA, 2015) & $\begin{array}{l}\text { "o uso de elementos de vídeojogos em contexto não-jogos para } \\
\text { melhorar a experiência do usuário e envolvimento do usuário" } \\
\text { (ZICHERMANN; CUNNINGHAM, 2010). }\end{array}$ \\
\hline (KIRÁL'OVÁ, 2015) & $\begin{array}{l}\text { "é um processo que utiliza as melhores ideias de jogos, lealdade e } \\
\text { economia comportamental para envolver as pessoas e motivá-las } \\
\text { a mudar de comportamento, desenvolver habilidades ou resolver } \\
\text { problemas" (ZICHERMANN; LINDER, 2013; BURKE, 2014). }\end{array}$ \\
\hline $\begin{array}{l}\text { (BÁRCENA; SANFILIPPO, } \\
\text { 2015) }\end{array}$ & $\begin{array}{l}\text { "o conceito de gamificação está relacionado com a utilização de } \\
\text { mecânicas próprias do jogo fora desses contextos, de modo que } \\
\text { as pessoas adotem, nesses contextos, aspectos positivos dos } \\
\text { jogos relacionados com o comportamento" (POPKIN, 2010). }\end{array}$ \\
\hline
\end{tabular}

(continua...)

2. Termo oriundo do inglês, sem tradução para português. Entretanto, é utilizado no texto no sentido de reconhecimento. 
Quadro 5 - Continuação

\begin{tabular}{l|l|}
\hline Autores & Definições \\
\hline $\begin{array}{l}\text { (YAMAMOTO; YOSHIN; } \\
\text { SONEHARA, 2015) }\end{array}$ & $\begin{array}{l}\text { "uso de elementos de design do jogo em contextos fora do jogo" } \\
\text { (DETERDING et al., 2011b). }\end{array}$ \\
\hline (DÍAZ et al., 2014) & $\begin{array}{l}\text { "uso de elementos de design do jogo em contextos não-jogo" } \\
\text { (DETERDING et al., 2011a). }\end{array}$ \\
\hline (GORDILLO et al., 2013) & $\begin{array}{l}\text { "uso de elementos de design do jogo em contextos não-jogo" } \\
\text { (DETERDING et al., 2011a). }\end{array}$ \\
\hline (GIOVANNELLA et al., 2013) & $\begin{array}{l}\text { "Uso de elementos de design do jogo em contextos não-jogo" } \\
\text { (DETERDING et al., 2011a). }\end{array}$
\end{tabular}

Fonte - Elaborado pelas autoras

Dos onze artigos analisados sobre a gamificação aplicada no setor do turismo, observa-se o uso predominante do conceito mais antigo desenvolvido pelos autores Deterding et al. (2011a). No entanto, as novas abordagens (BURKE, 2014; POPKIN, 2010; USKOV; SEKAR, 2014; ZICHERMANN; LINDER, 2013) apresentadas nos estudos de Bárcena e Sanfilippo (2015), Királ'ová (2015), Kachniewska (2015) e Negruşa et al. (2015), estão direcionadas ao conceito mais recente de Chou (2015), que centraliza-se na customização da aplicabilidade da gamificação e em suas limitações internas e externas.

\section{Autores e publicações que mais se destacam}

Ao considerar os 1.496 documentos, os autores com maior número de publicações são Hamari, Nakajima e Herzig. No que diz respeito à quantidade de publicação desses autores, o primeiro e o segundo apresentam-se com um total de treze documentos, e o terceiro com dez (Figura 4).

Figura 4 - Número de documentos publicados por autor

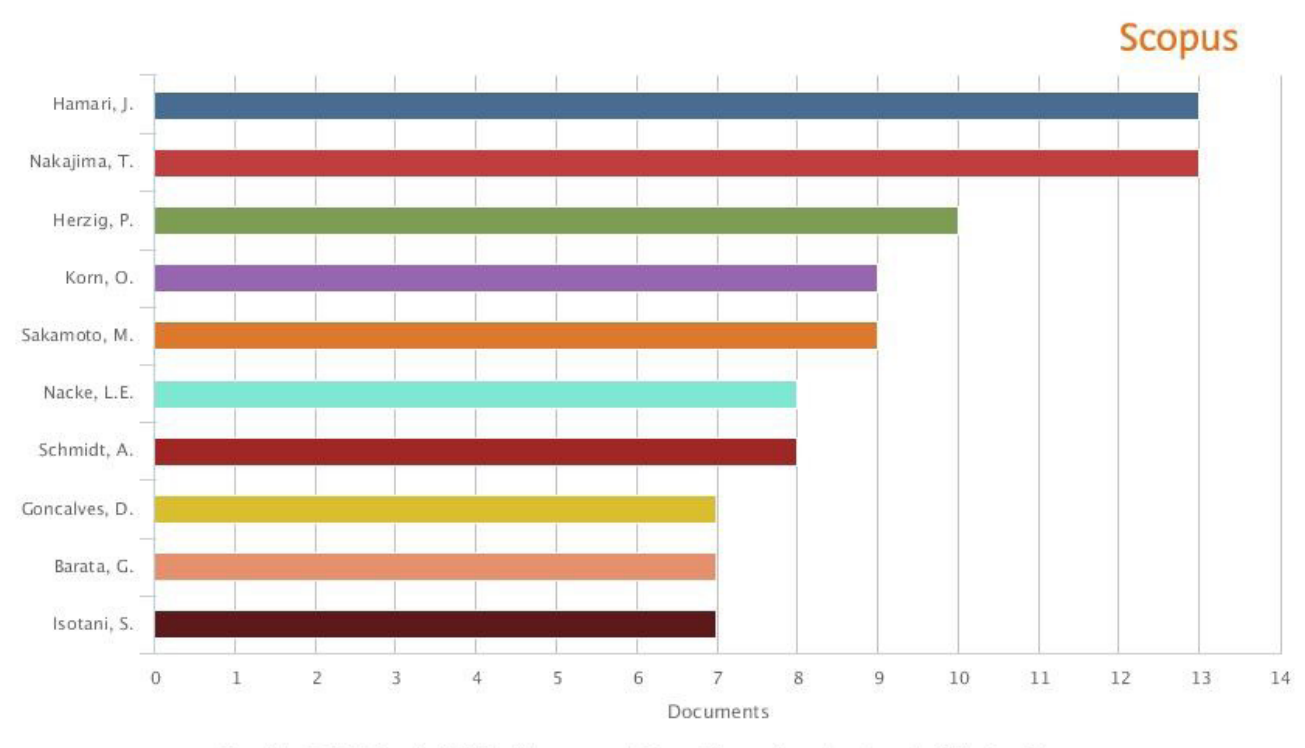

Fonte - Scopus (2016) 
Os artigos mais citados são os de Deterding et al. (2011a) (404), Deterding (2011b) (158), Domínguez et al. (2013) (157). Relativamente aos estudos sobre turismo, o autor que sobressai é Sigala (2015a, 2015b), com dois artigos. Os mais citados são os de Gordillo et al. (2013), com cinco artigos, e Sigala (2015a, 2015b), com quatro artigos.

A base de dados analisada expõe que a maior parte dos estudos sobre gamificação advém dos Estados Unidos (312 documentos), seguidos da Alemanha (145) e Inglaterra (127), e outros. No âmbito do turismo, as publicações de maior expressão vêm de países como Grécia (2), Espanha (1), República Tcheca (1) e outros. As áreas de pesquisa sobre a gamificação nos onze artigos aplicados ao turismo concentram um número maior de estudos na área da ciência da computação (6), e a segunda maior área de estudo, ainda no âmbito do turismo, são negócios, gestão e contabilidade (4).

\section{Objetivos, contextos e abordagens da gamificação no setor do turismo}

Inúmeras são as possibilidades de aplicação da gamificação no setor de turismo. Este artigo identificou essas aplicabilidades (Quadro 6). Além de perceber os objetivos que os estudos pretenderam atingir, fez-se necessário apontar o campo de abordagens teóricas e contextos que os estudos priorizaram.

Os estudos de componente teórica possuem diferentes apontamentos entre si, e seus objetivos são descritos para que haja um melhor entendimento sobre suas abordagens. Gordillo et al., (2013) propõem um modelo genérico para apoiar uma nova maneira de visitar uma cidade. Baseando-se numa revisão de literatura, os autores definiram um modelo de passeio educativo, considerando três dimensões: turismo, aprendizagem e gamificação. Nesse estudo, o turismo é considerado o ponto de partida das atividades direcionadas à aprendizagem, e a gamificação corresponde aos elementos contidos na aplicação mobile, como emblemas, painel de pontuação e sistemas ganhos.

Quadro 6 - Objetivos, contextos e abordagens da gamificação no setor do turismo

\begin{tabular}{|c|c|c|c|}
\hline Objetivo & $\begin{array}{c}\text { Contexto de } \\
\text { aplicação }\end{array}$ & Abordagens teóricas & Autor/ano \\
\hline $\begin{array}{l}\text { Explorar a tendência } \\
\text { da gamificação e } \\
\text { seu potencial para } \\
\text { o desenvolvimento } \\
\text { de experiências } \\
\text { e marketing no } \\
\text { turismo. }\end{array}$ & Marketing & $\begin{array}{l}\text {-Conceito de game } \\
\text {-Motivações para a prática } \\
\text { de jogos } \\
\text {-Game e turismo } \\
\text {-Gamificação e o marketing } \\
\text { turístico }\end{array}$ & (XU et al., 2015) \\
\hline $\begin{array}{l}\text { Investigar o uso } \\
\text { e os impactos da } \\
\text { gamificação em um } \\
\text { contexto específico } \\
\text { do turismo } \\
\text { (e-commerce/ } \\
\text { TripAdvisor). }\end{array}$ & $\begin{array}{c}\text { Plataforma de } \\
\text { planejamento } \\
\text { de viagem e } \\
\text { avaliações online }\end{array}$ & $\begin{array}{l}\text {-Definição de gamificação e } \\
\text { Funware } \\
\text {-Resultados psicológicos e } \\
\text { comportamentais eficazes } \\
\text {-Contexto do estudo e } \\
\text { questões de pesquisa } \\
\text {-Funware design: affordances } \\
\text { motivacionais e valores } \\
\text { experimentais }\end{array}$ & (SIGALA, 2015b) \\
\hline
\end{tabular}


Quadrro 6 - Continuação

\begin{tabular}{|c|c|c|c|}
\hline Objetivo & $\begin{array}{l}\text { Contexto de } \\
\text { aplicação }\end{array}$ & Abordagens teóricas & Autor/ano \\
\hline $\begin{array}{l}\text { Identificar técnicas } \\
\text { da gamificação e } \\
\text { suas aplicações } \\
\text { utilizadas pelas } \\
\text { organizações } \\
\text { na indústria da } \\
\text { hospitalidade } \\
\text { e do turismo } \\
\text { para melhorar } \\
\text { suas atividades } \\
\text { sustentáveis. }\end{array}$ & Sustentabilidade & $\begin{array}{l}\text { - Conceito de gamificação } \\
\text { - Contextos de aplicação } \\
\text {-Gamificação e } \\
\text { sustentabilidade }\end{array}$ & $\begin{array}{c}\text { (NEGRUŞA et al., } \\
\text { 2015) }\end{array}$ \\
\hline $\begin{array}{l}\text { Revê a literatura } \\
\text { para identificar } \\
\text { os princípios e } \\
\text { elementos do jogo } \\
\text { que podem levar a } \\
\text { uma gamificação } \\
\text { eficaz. }\end{array}$ & Marketing & $\begin{array}{l}\text { - Gamificação em Marketing: } \\
\text { conceito, aplicações e } \\
\text { benefícios } \\
\text { - Implementando a } \\
\text { gamificação: elementos e } \\
\text { princípios de funware design } \\
\text { - Aplicação de gamificação } \\
\text { em marketing } \\
\text { - Antes da compra / } \\
\text { consumo: experiência } \\
\text { turismo } \\
\text { - Durante a compra / } \\
\text { consumo: experiência } \\
\text { turismo } \\
\text { - Após a compra / consumo: } \\
\text { experiência do turismo } \\
\text { experiência }\end{array}$ & (SIGALA, 2015a) \\
\hline $\begin{array}{l}\text { Expor a ideia de } \\
\text { uma nova aplicação } \\
\text { mobile, que } \\
\text { integrasse jogos } \\
\text { sociais e tecnologia } \\
\text { baseada em } \\
\text { localização, podendo } \\
\text { levar ao interesse } \\
\text { crescente da rede } \\
\text { social do marketing } \\
\text { no turismo baseado } \\
\text { na localização. }\end{array}$ & Aplicação mobile & $\begin{array}{l}\text { - Gamificação e mídias } \\
\text { sociais como uma ferramenta } \\
\text { de marketing } \\
\text { - Design do game e suas } \\
\text { técnicas no turismo }\end{array}$ & $\begin{array}{c}\text { (KACHNIEWSKA, } \\
2015)\end{array}$ \\
\hline $\begin{array}{l}\text { Descrever como a } \\
\text { gamificação pode } \\
\text { ser usada para } \\
\text { atrair a atenção } \\
\text { dos visitantes, } \\
\text { despertando o } \\
\text { interesse e gerando } \\
\text { o comportamento } \\
\text { desejado - visitar } \\
\text { um determinado } \\
\text { destino. }\end{array}$ & $\begin{array}{c}\text { Marketing do } \\
\text { destino turístico }\end{array}$ & $\begin{array}{l}\text { - O Homo visitante } \\
\text { transformado em Homo } \\
\text { Ludens? } \\
\text { - A gamificação e o } \\
\text { marketing de destino } \\
\text { turístico }\end{array}$ & (KIRÁL'OVÁ, 2015) \\
\hline
\end{tabular}

(continua...) 


\section{Quadro 6 - Continuação}

\begin{tabular}{|c|c|c|c|}
\hline Objetivo & $\begin{array}{l}\text { Contexto de } \\
\text { aplicação }\end{array}$ & Abordagens teóricas & Autor/ano \\
\hline $\begin{array}{l}\text { Identificar o valor } \\
\text { dos recursos } \\
\text { pedagógicos lúdicos } \\
\text { na aprendizagem } \\
\text { de línguas num } \\
\text { contexto digital. }\end{array}$ & Educação - línguas & $\begin{array}{l}\text { - Gamificação } \\
\text { - Jogos } \\
\text { - Aprendizagem }\end{array}$ & $\begin{array}{l}\text { (BÁRCENA; } \\
\text { SANFILIPPO, } \\
\text { 2015) }\end{array}$ \\
\hline $\begin{array}{l}\text { Desenvolver o } \\
\text { sistema de coleta } \\
\text { "Photopolie" } \\
\text { foto digital que } \\
\text { inclui informação } \\
\text { geográfica. }\end{array}$ & Mídias digitais & $\begin{array}{l}\text { - Esboço da figura } \\
\text { Photopolie } \\
\text { - Funções do Photopolie } \\
\text {-Função, localização, } \\
\text { aquisição, pontuação de } \\
\text { submissão de fotos digitais }\end{array}$ & $\begin{array}{c}\text { (YAMAMOTO, } \\
\text { YOSHIN; } \\
\text { SONEHARA, 2015) }\end{array}$ \\
\hline $\begin{array}{l}\text { Relatar a utilização } \\
\text { de terminais } \\
\text { móveis em espaços } \\
\text { históricos para } \\
\text { realização de um } \\
\text { jogo de aventura, } \\
\text { utilizando uma } \\
\text { plataforma baseada } \\
\text { em localização para } \\
\text { despertar a fantasia } \\
\text { e curiosidade das } \\
\text { crianças sobre o } \\
\text { patrimônio cultural. }\end{array}$ & $\begin{array}{l}\text { Terminais móveis } \\
\text { em lugares } \\
\text { históricos para } \\
\text { realização um jogo } \\
\text { de aventura }\end{array}$ & $\begin{array}{l}\text { - A ideia de desenvolver um } \\
\text { App } \\
\text {-Por que pervasive games são } \\
\text { adequados para ambientes } \\
\text { culturais? } \\
\text { - Adaptação de elementos } \\
\text { históricos a um ambiente } \\
\text { contemporâneo } \\
\text { - Narrativa em transmídia } \\
\text { - Equilíbrio entre jogadores } \\
\text { e a história }\end{array}$ & (DÍAS et al., 2014) \\
\hline $\begin{array}{l}\text { Propor um modelo } \\
\text { genérico para apoiar } \\
\text { uma nova maneira } \\
\text { de visitar uma } \\
\text { cidade. }\end{array}$ & $\begin{array}{l}\text { Rotas educacionais } \\
\text { / aplicação mobile }\end{array}$ & $\begin{array}{l}\text { - Formal aprendizagem ao } \\
\text { ar livre } \\
\text { - Informal de Aprendizagem } \\
\text { ao ar livre } \\
\text { - Requisitos } \\
\text { - Estrutura passeio } \\
\text { educativo }\end{array}$ & $\begin{array}{l}\text { (GORDILLO et al., } \\
\text { 2013) }\end{array}$ \\
\hline $\begin{array}{l}\text { Propor soluções } \\
\text { "glocais" para o } \\
\text { acesso à informação } \\
\text { e participação dos } \\
\text { locais nos processos } \\
\text { de aprendizagem } \\
\text { informais } \\
\text { localizados em uma } \\
\text { smart city. }\end{array}$ & $\begin{array}{l}\text { Smart city e } \\
\text { aprendizagem }\end{array}$ & $\begin{array}{l}\text { - Smart city e os modelos } \\
\text { adotados } \\
\text { - O caso de estudo: Villard- } \\
\text { de-Lans } \\
\text { - Gamificação }\end{array}$ & $\begin{array}{l}\text { (GIOVANNELLA } \\
\text { et al., 2013) }\end{array}$ \\
\hline
\end{tabular}

\section{Fonte - Elaborado pelas autoras}

Negruşa et al. (2015) identificaram técnicas de gamificação e aplicações utilizadas pelas organizações na indústria da hospitalidade e do turismo, para melhoria de suas atividades sustentáveis. Os autores fundamentaram-se em uma pesquisa detalhada da indústria, por meio de estudos de caso autoidentificados de aplicações gamificadas no setor do turismo e hospitalidade. 
Kachniewska (2015) expõe a ideia de uma nova aplicação mobile, que integra jogos "sociais" e tecnologia baseada em localização, o que pode levar ao interesse crescente da rede social e do marketing baseado na localização. A autora alicerçou-se em uma profunda revisão de literatura sobre gamificação e apresentou uma visão ampla sobre a oportunidade do uso de mídia social no campo da promoção do turismo. Além disso, são apresentados os objetivos de implementação da gamificação, sendo eles: gerar engajamento, mudar o comportamento e estimular a inovação. Para além desses, as técnicas de gamificação pretendidas no estudo buscavam aumentar os desejos naturais das pessoas para a competição, realização, status, autoexpressão e altruísmo.

Sigala (2015a) reviu a literatura para identificar os elementos e princípios do jogo que podem levar a uma gamificação eficaz e que, em seguida, demonstrou a aplicabilidade e as implicações dessa teoria, discutindo várias aplicações gamificadas desenvolvidas para apoiar práticas de marketing e crowdsourcing no turismo. Todos os objetivos propostos por Gordillo et al. (2013); Negruşa et al. (2015); Kachniewska (2015); Sigala, (2015a), foram atingidos a partir de uma revisão teórica multidisciplinar, alcançando temas do marketing, sustentabilidade, educação, mobilidade e tecnologia.

Os estudos trazem o foco da abordagem teórica no marketing (KIRÁL'OVÁ, 2015; SIGALA, 2015a; XU et al., 2015). Xu et al. (2015), a partir da revisão de literatura, apresentam um gráfico de curva linear crescente para expor as motivações do uso do mobile game por diferentes grupos de usuários (turistas e não turistas). As motivações para o jogo pelos não turistas são: divertimento, passar o tempo, parentesco, socialização. Os intermediários são motivados por: tempo limitado, pouco familiarizado com o ambiente, ansioso para conhecer o lugar. Já os turistas são motivados por: ser interativo, fantasia, limite de tempo, pouco familiarizado com o ambiente, divertimento. Já Sigala (2015a) observou as motivações sob a perspectiva do modelo de negócios gamificado da TripAdvisor. A autora apresenta que as motivações reconhecidas e os valores experimentais se externalizam através da competência (realização e concorrência), socialização, relações, autonomia, relacionamento e mecanismo para aumentar o status. Entender as motivações pelas quais os turistas se interessam pelo ato de jogar ou por apps gamificados contribui para uma customização da aplicação da gamificação em diferentes cenários.

No contexto da educação e aprendizagem, a gamificação é utilizada com o propósito de motivar e estimular os alunos, inclusive da disciplina de línguas do curso de licenciatura em turismo como apresenta o estudo de Bárcena e Sanfilippo (2015). Algumas atividades de estímulo a aprendizagem podem ser praticadas, por exemplo, assistir a um vídeo sobre o lugar visitado, um jogo de quebra-cabeça que desafia a inteligência do aluno (GORDILLO et al., 2013). A gamificação, quando utilizada para fins educacionais, contribui para a predisposição natural do ser humano para a competição, a fim de amenizar determinadas tarefas e envolver os usuários (BÁRCENA; SANFILIPPO, 2015).

Um conceito que abrange pragmaticamente as dimensões aprendizagem, gamificação e turismo é abordado por Giovanella et al. (2013), que sugere uma aprendizagem a partir de uma smart city. Nesse estudo, a gamificação entra em uma terceira etapa que envolve os jogadores (turistas/visitantes) em atividades colaborativas através de pontos de interação. 
O uso da gamificação na conjuntura da sustentabilidade é abordado por Negruşa et al. (2015) que afirmam que "a gamificação tem o potencial para melhorar a sustentabilidade do turismo", havendo, inclusive, possíveis compradores de serviços gamificados na área, sendo eles: empresas (cadeias de hotéis, restaurantes, operadores turísticos), instituições locais (escritório do prefeito, órgãos oficiais de turismo, escritórios públicos de emprego) e ONG (preocupado com social, ambiental e as questões políticas). Ademais, os autores apresentam três frameworks com os benefícios e efeitos (ambiental, social e econômico) da gamificação no relacionamento das organizações de turismo sob o ponto de vista dos empregados, turistas e comunidade.

\section{Potencial da gamificação para a competitividade no turismo}

Os turistas estão atentos ao valor agregado dos produtos e serviços, baseando-se em uma suposição de certa combinação de uma experiência holística. Esses turistas tornaram-se resistentes a anúncios publicitários de massa e passaram a ser muito individualistas. Como consequência, os destinos começaram a se concentrar na construção de parcerias de apoio ao desenvolvimento de um destino turístico competitivo (KACHNIEWSKA, 2015). A introdução de novas tecnologias nessa dinâmica competitiva contribuiu para a concepção da gamificação como ferramenta de apoio para diferenciar produtos e serviços.

Smartphones, tablets, phablets e aplicações móveis têm uma importante relevância para os visitantes (KIRÁL'OVÁ, 2015). 0 advento das tecnologias móveis, ou seja, toda tecnologia que permite seu uso durante a movimentação do usuário, trouxe a oportunidade de jogar em todos os lugares (WEBER, 2014). 0 uso destes jogos oferece uma variedade de benefícios para a comercialização do turismo e pode aumentar a notoriedade da marca, atrair potenciais clientes, melhorar as experiências in loco dos turistas e aumentar o engajamento (XU et al., 2015).

Hoje, elementos de mecânica de jogos estão sendo empregado em diversas áreas como educação, esfera militar, treinamento corporativo, marketing, ecologia ambiental, campanhas de políticas públicas, programas de exercícios físicos (KACHNIEWSKA, 2015) e turismo. No último caso, a disseminação ocorreu após o sucesso do Foursquare ${ }^{3}$, que oferece pontos e troféus de acordo com a utilização do aplicativo, como uma forma de motivação e retenção dos usuários. Os interesses das empresas sobre o uso da gamificação surgiram devido a sua capacidade de revolucionar a interação humano-computador e a experiência do usuário (SIGALA, 2015a). As oportunidades de negócios são diversas: clientes mais engajados, crowdsourcing e inovação para melhorar o desempenho do empregado (KACHNIEWSKA, 2015).

No contexto dos destinos turísticos há diversas formas de gamificação, geralmente utilizadas como transmídia, letterboxing ${ }^{4}$, busca geocaching ${ }^{5}$, waymarking ${ }^{6}$,

3. Foursquare - serviço turístico desenvolvido pela empresa de mesmo nome que utiliza a tecnologia para localização inteligente a fim de criar experiências de consumo e soluções de negócios significativas. Ver mais detalhes em: https://pt.foursquare.com/.

4. É um hobby ao ar livre que combina elementos de orientação, arte e quebra-cabeças. Visto em: http://bit.ly/2n0VemU.

5. É um tipo de "caça ao tesouro" que se utiliza de GPS.

6. É um meio pelo qual as pessoas podem catalogar, marcar, localizar lugares do mundo através do GPS. Visto em: http://bit.ly/2n6MVaM. 
caça ao tesouro, wherigo $^{7}$, resposta rápida, e realidade aumentada (KIRÁL'OVÁ, 2015). No entanto, nos documentos analisados foram encontradas maneiras adicionais de utilização da gamificação como ferramenta de competitividade nos destinos turísticos, como guia baseado em geolocalização, promoção da sustentabilidade ambiental e social, e uploading de fotografias tiradas durante as viagens.

Algumas influências positivas da gamificação em destinos turísticos (Quadro 7) são evidenciadas para justificar a ferramenta como uma possível aliada para o aumento da competitividade nos destinos turísticos. Em uma smart city, a gamificação pode aumentar a conscientização sobre o consumo de recursos e ser estendida a muitos aspectos relevantes como (governança inteligente, e-democracia etc.) para promover, por exemplo, a aquisição de alguns dos chamados soft skills do século XXI como tomada de decisão, liderança e equipe de trabalho (GIOVANNELLA et al., 2013). Além disso, várias novas oportunidades de aprendizagem podem ser alcançadas a partir de atividades ao ar livre, fazendo o uso da M-Learning (aprendizagem via mobile) formal e informal, usando a cidade como uma plataforma de aprendizagem em combinação com a gamificação (GORDILLO et al., 2013).

Quadro 7 - Influências positivas da gamificação nos destinos turísticos

\begin{tabular}{|l|l|}
\hline Autor/ano & Influências positivas da gamificação nos destinos \\
\hline Giovannella et al. (2013) & $\begin{array}{l}\text { Aquisição de alguns dos chamados soft skills (tomada de decisão, } \\
\text { liderança e trabalho em equipe) }\end{array}$ \\
\hline Gordillo et al. (2013). & Aprendizagem via mobile \\
\hline Kachniewska (2015) & Promoção do turismo \\
\hline Negruşa et al. (2015) & Descobrir a história, a vida e a identidade dos destinos \\
\hline Sigala (2015b). & $\begin{array}{l}\text { Os processos de formação e o impacto à imagem de destinos/ } \\
\text { empresas, formação e difusão de modas e tendências na indústria } \\
\text { do turismo }\end{array}$ \\
\hline Királ'ová (2015) & $\begin{array}{l}\text { Experimentar novas atrações e atividades de lazer, vivenciando um } \\
\text { destino singular, aumento de visitação, envolvimento dos visitantes } \\
\text { e extensão de sua estadia, engajamento e lealdade }\end{array}$ \\
\hline & Fonte - Elaborado pelas autoras \\
\hline
\end{tabular}

Além do mais, a técnica de gamificação pode ser utilizada na promoção de destinos, por intermédio de uma aplicação mobile. Citando caso análogo, o app "Play Mazovia" sugerido no estudo de Kachniewska (2015) pode ser necessário por dois propósitos: um deles cobre as necessidades de entidades de turismo por meio da promoção do destino, enquanto o segundo contribui para a promoção do turismo a partir da prática de atividade desportiva entre os adolescentes no destino.

Por meio do uso de novos elementos incorporados a produtos e serviços gamificados, o turismo poderá tomar proveito de objetivos econômicos com externalidades sociais e ambientais positivas. Além de outras possibilidades, como o ambiente de treinamento tornando-se mais envolvente, e a oportunidade dos turistas tomarem conhecimento da história, da vida e da identidade dos destinos,

7. É uma plataforma que reconhece o local por GPS, que combina um jogo de aventura e uma geocache. Visto em https://en.wikipedia.org/wiki/Wherigo. 
todos esses com um impacto negativo mínimo no local, comunidade e o meio ambiente (NEGRUŞA et al., 2015).

As empresas de turismo e gestores de destinos podem participar no contexto da gamificação para colaborar ativamente com os viajantes em várias atividades de cocriação, a fim de influenciar as experiências de viagem dos turistas, os processos de formação e impactar a imagem de destinos/empresas, e na formação e difusão de modas e tendências na indústria do turismo (SIGALA, 2015b).

Ambos os visitantes e os destinos podem ganhar aplicando a gamificação em marketing de destino. Visitantes ganham por experimentar novas atrações e atividades de lazer, vivenciando um destino singular. 0 destino ganha pelo aumento de visitação, envolvimento dos visitantes, estendendo sua estadia e motivando o seu engajamento e lealdade (KIRÁL'OVÁ, 2015).

Na próxima seção, serão expostas as abordagens metodológicas adotadas nos estudos em análise.

\section{Abordagens metodológicas adotadas nos estudos analisados}

Os artigos analisados apresentam uma pequena diferença entre suas abordagens. Para uma percepção sistemática, o Quadro 8 expõe a divisão dos artigos quanto ao tipo de abordagem (empírica e teórica).

Quadro 8 - Classificação dos estudos quanto à natureza do trabalho

\begin{tabular}{l|l|}
\hline Empíricos & Teóricos \\
\hline $\begin{array}{l}\text { Giovannella et al. (2013); Díaz, Toftedahl e } \\
\text { Svensson (2014); Sigala (2015b); Xu et al. (2015); } \\
\text { Bárcena e Sanfilippo (2015); Yamamoto, Yoshin e } \\
\text { Sonehara (2015); Királ'ová (2015) }\end{array}$ & $\begin{array}{l}\text { Kachniewska (2015); Sigala (2015a) } \\
\text { (2015); }\end{array}$ \\
\hline
\end{tabular}

Fonte - Elaborado pelas autoras

Os estudos enquadrados como empíricos foram divididos em qualitativos e quantitativos, e também categorizados quanto à coleta e análise dos dados (quadros 9 e 10). Por se tratar de uma área nova, exploratória e emergente no setor do turismo, a gamificação precisa ser explorada em profundidade, sendo assim, o paradigma de pesquisa qualitativa se faz coerente em alguns estudos.

Para atingir o objetivo de "propor soluções glocais ${ }^{8 "}$ para o acesso à informação e participação dos locais nos processos de aprendizagem informais localizados em uma smart city, Giovannella et al. (2013) desenvolveram um estudo de caso na Villard-de-Lans, nos Alpes franceses, composta por sete centros residenciais. 0 estudo recorre à entrevista estruturada com os residentes da comunidade em estudo. Os estudos de Bárcena e Sanfilippo (2015) e Királ’ová (2015) também aplicaram entrevista estruturada como método de coleta de dados.

No seu estudo aplicado às crianças de um bairro chamado Skövde, na Suécia, Díaz, Toftedahl e Svensson (2014) relataram a utilização de terminais móveis em espaços históricos para a prática de um jogo de aventura, utilizando uma plataforma baseada em localização para despertar a fantasia e curiosidade das crianças sobre o patrimô-

8. Glocais: termo utilizado pelos autores Giovannella et al. (2013) como junção de globais e locais. 
nio cultural. Os autores dividiram a recolha de dados em duas fases: qualitativa (entrevista semiestruturada e observação sistemática), e quantitativa (questionários). Foram inquiridas 82 crianças de quatro classes de duas escolas diferentes do bairro. 0 uso de um ou mais métodos de coleta e análise de dados contribuem para um design minucioso e permitiu uma validação mais completa dos resultados.

Quadro 9 - Metodologia para coleta de dados nos estudos empíricos

\begin{tabular}{|c|c|}
\hline Metodologia de coleta de dados & Estudos empíricos \\
\hline $\begin{array}{l}\text { Qualitativo } \\
\text { Entrevistas estruturadas } \\
\text { Entrevista semiestruturada e observação } \\
\text { sistemática } \\
\text { focus group }\end{array}$ & $\begin{array}{l}\text { Giovannella et al., 2013; Bárcena e Sanfilippo, } \\
\text { 2015; Királ'ová, 2015; Xu et al. (2015) } \\
\text { Díaz, Toftedahl e Svensson, } 2014 \\
\text { Xu et al (2015) }\end{array}$ \\
\hline $\begin{array}{l}\text { Quantitativo } \\
\text { Questionário }\end{array}$ & $\begin{array}{l}\text { Díaz, Toftedahl e Svensson (2014); Sigala } \\
\text { (2015b); Yamamoto, Yoshin e Sonehara (2015); } \\
\text { Bárcena e Sanfilippo (2015) }\end{array}$ \\
\hline
\end{tabular}

Fonte - Elaborado pelas autoras

O estudo de Xu et al. (2015) foi o único a utilizar o focus group como meio de recolha de dados. A aplicação do método se explica, pois geralmente é utilizado para temas que não são bem compreendidos e para descobrir novos insights (XU et al., 2015), como é o caso desta pesquisa. Para se inserirem no estudo, os respondentes tinham que obedecer aos seguintes critérios: (1) Usar smartphone com frequência; (2) jogar jogos de PC ou jogos móveis pelo menos duas vezes por semana; (3) feito pelo menos uma viagem de férias durante os últimos doze meses. No total se enquadraram nos critérios 26 estudantes voluntários. Estabeleceram-se quatro grupos de estudantes a partir das categorias (grau de estudo, sexo, idade e status de material).

No tocante da coleta de dados, em termos quantitativos, o questionário é o instrumento mais corrente. Esses questionários foram aplicados com Yamamoto, Yoshin e Sonehara (2015) - doze estudantes de ciência da informação, Díaz, Toftedahl e Svensson (2014) - 82 crianças, Bárcena e Sanfilippo (2015) - 273 graduandos de turismo do curso de línguas, e Sigala (2015) - 3.137 viajantes. 0 estudo de Sigala (2015) possuiu mais representatividade em número de inquiridos, por isso, talvez fosse o único estudo a utilizar análises multivariadas.

Quadro 10 - Metodologia para análise de dados nos estudos empíricos

\begin{tabular}{|l|l|}
\hline Metodologia para análise de dados & Estudos empíricos \\
\hline $\begin{array}{l}\text { Qualitativo } \\
\text { Análise de conteúdo }\end{array}$ & Giovannella et al. (2013); Guadalupe, et al. \\
Análise temática & (2014); Bárcena e Sanfilippo (2015) \\
Quantitativo & Xu et al. (2015) \\
$\begin{array}{l}\text { Estatística descritiva } \\
\text { Qui-quadrado, Teste-T, Análise Fatorial } \\
\text { Exploratória (EFA) }\end{array}$ & Bárcena e Sanfilippo (2015); Yamamoto, Yoshin \\
& e Sonehara (2015) \\
\hline & Sigala (2015b) \\
\hline
\end{tabular}

Na análise de dados, estatística descritiva, qui-quadrado, Teste-T, Análise Fatorial Exploratória (EFA), são habitualmente utilizados em dados quantitativos 
e foram identificados nos estudos analisados. Nos estudos qualitativos, a análise de conteúdo e a análise temática foram os métodos encontrados (Quadro 9). Diante do cenário apresentado, conclui-se que a maior parte dos estudos é de caráter qualitativo, com método de coleta de dados mais comum - entrevistas e com análise de conteúdo como forma de análise de dados.

\section{CONCLUSÕES, IMPLICAÇÕES PARA FUTURAS PESQUISAS E LIMITAÇÕES}

O principal objetivo deste artigo foi realizar uma revisão de literatura acerca do tema gamificação e turismo, a fim de elencar indicações nos estudos que justificassem o potencial da gamificação para a elevação da competitividade nos destinos turísticos. Com base na revisão exploratória de literatura, se extraiu conclusões, limitações e caminhos para futuros estudos.

Baseando-se em artigos precursores, alguns estudos posteriores foram desenvolvidos no âmbito do turismo (BÁRCENA; SANFILIPPO, 2015; DÍAZ; TOFTEDAHL; SVENSSON, 2014; GIOVANNELLA et al., 2013; GORDILLO et al., 2013; KACHNIEWSKA, 2015; KIRÁL'OVÁ, 2015; NEGRUŞA et al., 2015; SIGALA, 2015a, 2015b; XU et al., 2015; YAMAMOTO; YOSHIN; SONEHARA, 2015, entre outros), os quais versaram sobre smart city e o design da aprendizagem, destinos turísticos, promoção da sustentabilidade, experiências turísticas, aplicações mobile para turismo e sistemas digitais de fotografias no turismo (Quadro 3).

Entretanto, a partir dos onze artigos analisados, conclui-se que a gamificação como ferramenta direcionada à competitividade dos destinos turísticos é investigada por poucos autores, por exemplo, Kachniewska (2015) e Királ'ová (2015). Esses estudos apresentam duas principais conclusões: os visitantes estão mais abertos às novas experiências e desafios durante suas viagens, especialmente quando conectados com a diversão. E a gamificação pode ser utilizada como uma ferramenta eficaz para a promoção dos destinos através de aplicações mobile.

Notou-se que, apesar da recente discussão sobre o uso da ferramenta, a gamificação já é considerada uma nova abordagem para promover os destinos turísticos. Além disso, fornece para as organizações de turismo e marketing uma oportunidade de criar configurações informativas com entretenimento para a consciência bem-sucedida da marca, interação e comunicação do turista com o destino (XU et al., 2015).

A partir das análises dos onze documentos expostas durante o curso deste artigo, pode-se concluir uma incipiente síntese do conhecimento e apresentar o que é sabido sobre gamificação, como conceitos, principais autores, evolução do conceito no tempo, principais campos de aplicação, quantidade de documentos publicados na base do Scopus por ano, principais países de publicação sobre o tema; e o que se conhece sobre gamificação aplicada nos destinos turísticos, que compreende os principais autores, situações práticas, estudos relevantes sobre o tema, exemplos específicos de aplicação da gamificação nos destinos turísticos e as principais abordagens adotadas pelos autores. Outrossim, com este estudo consegue-se projetar o que ainda é preciso saber sobre gamificação (efeitos da gamificação no comportamento do consumidor, princi- 
pais ferramentas que, aliadas à gamificação, podem aumentar a competitividade, entre outros) e sobre gamificação aplicada nos destinos turísticos (implicações a nível monetário ao introduzir a tecnologia em destinos turísticos, se a gamificação contribui para o aumento da permanência de turistas no destinos, impacto emocional da gamificação nos turistas, níveis de envolvimento e motivação proporcionados pela gamificação, e outros).

Levando-se em consideração os aspectos apresentados na síntese do conhecimento e em virtude dos dados apresentados sobre o número de documentos encontrados na base de dados Scopus, é evidente que as pesquisas sobre a temática estão em ascensão; assim como a curva de interesse pelo tema da gamificação por parte de pesquisadores, oferta e demanda no setor do turismo, também se encontra em status positivo. Entretanto, apesar do interesse, ainda é reduzido o número de estudos, dificultando análises profundas e comparativas.

A maior parte dos estudos analisados, independentemente do contexto e abordagem, conjecturou a gamificação apenas sob os fatores positivos (envolvimento, motivação, emoção etc.). Alguns fatores negativos (limitar a ferramenta a um sistema de pontos e medalhas, focar apenas no virtual e esquecer o mundo real, não personalização do público-alvo, entre outros) não foram amplamente contemplados. Também pode-se inferir que, apesar do estágio de exploração em que o tema ainda se encontra, em alguns casos, a abordagem qualitativa se faz necessária. Entretanto, isso não justifica que a maioria dos estudos tenham negligenciado ênfases quantitativas, impedindo algumas generalizações de resultados.

Em vista dos argumentos apresentados, a partir da revisão de literatura, nomeadamente na seção sobre o potencial da gamificação como geradora da competitividade nos destinos turísticos, identificam-se grupos de estudos que se situam nos campos da aprendizagem, sustentabilidade, mobilidade, tecnologia e marketing. Ao levar em consideração esses aspectos, conclui-se que, para que um destino turístico consiga aumentar sua competitividade utilizando-se da gamificação como ferramenta para este fim, sugere-se que os destinos turísticos ampliem suas utilizações para setores antes não explorados, tais como mercado financeiro, varejo, economia criativa de pequenos negócios e outros, gerando valor para além dos turistas.

A escassez de estudos que abordam a gamificação sob uma perspectiva mais econômica, de certa forma limitou a obtenção de conclusões mais holísticas sobre o real potencial da gamificação para a competitividade dos destinos turísticos. Outra limitação identificada diz respeito à banalização do uso do termo gamification, dificultando a localização de pesquisas que trazem contributos fiáveis.

Em relação às pesquisas futuras, seria inovadora a realização de pesquisas mais abrangentes, como teses e dissertações, que tivessem como objetivo analisar de que maneira as empresas brasileiras vêm desenvolvendo produtos e serviços gamificados para o setor do turismo. Também, investigar até que ponto a gamificação impacta na experiência dos turistas durante a viagem. Além disso, seria cabível uma pesquisa mais ampla sobre a gamificação na perspectiva da sustentabilidade, que buscasse ir além da replicação de casos de sucesso sobre a gamificação no turismo, expondo uma abordagem mais recente e crítica sobre o potencial da gamificação para tornar o turismo mais sustentável.

De acordo com os exemplos, argumentos e tópicos que foram expostos no desenvolvimento deste trabalho, pode-se inferir que a introdução deste trabalho é 
de fato verdadeira, quando diz que o novo modelo de turismo tem foco na experiência obtida nos destinos pelos turistas, a fim de proporcioná-los, bem como aos visitantes, experiências impactantes, lúdicas e únicas. Diante da afirmação, este artigo ajudou a identificar a gamificação como uma ferramenta de alto potencial para alcance da competitividade nos destinos turísticos por meio de aplicações mobile, realidade aumentada, guias turístico-educacionais, oferecimento de "créditos de carbono" da oferta para a demanda, tendo em vista a promoção da sustentabilidade e outros benefícios.

\section{REFERÊNCIAS}

ASQUER, A.; KRACHKOVSKAYA, I. Can gamification assist the implementation of co-production tools? Some experimental evidence from the monitoring of the urban environment. In: INTERNATIONAL CONFERENCE ON PUBLIC POLICY, 2., 2015. Proceedings... Milan: ICPP, 2015.

BÁRCENA, E.; SANFILIPPO, M. La píldora informativa audiovisual como estrategia de gamificación en los cursos en línea de segundas lenguas. Círculo de Lingüística Aplicada a la Comunicación, Madrid, v. 63, p. 122-151, 2015.

BURKE, B. Gamify: how gamification motivates people to do extraordinary things. Brookline: Bibliomotion, 2014.

DETERDING; S. et al. From game design elements to gamefulness: defining "gamification", 2011a. In: INTERNATIONAL ACADEMIC MINDTREK CONFERENCE: ENVISIONING FUTURE MEDIA ENVIRONMENTS, 15., 2011, Tampere. Proceedings... Tampere: MindTrek, 2011a. p. 9-15. Disponível em: <http://bit.ly/1LgKAQX>. Acesso em: 24 mar. 2017.

DETERDING, S. et al. Gamification: using game-design elements in non-gaming contexts In: ANNUAL CONFERENCE EXTENDED ABSTRACTS ON HUMAN FACTORS IN COMPUTING SYSTEMS, 2011, Vancouver. Proceedings... Vancouver: CHI EA, 2011b. p. 2425-2428. Disponível em: <http://doi.org/10.1145/1979742.1979575>. Acesso em: 24 mar. 2017.

DÍAZ, M. G. A.; TOFTEDAHL, M.; SVENSSON, T. The Mystery of Elin: incorporating a city cultural program on history and heritage into a pervasive game. In: INTERACTIVE ENTERTAINMENT, 2014. Proceedings... Newcastle: ACM, 2014.

FERREIRA, A. F. M. Gamification: um novo paradigma de criação de valor no Mass Market. 2015. Dissertação (Mestrado em Gestão) -Universidade de Aveiro, Aveiro, 2015.

GIOVANNELLA, C. et al. Villard-de-Lans: a case study for participatory people-centered smart city learning design. In: IEEE INTERNATIONAL CONFERENCE ON ADVANCED LEARNING TECHNOLOGIES, 13., 2013. Proceedings... Beijing: IEEE, 2013.

GORDILLO, A. et al. The city as a learning gamified platform. In: FRONTIERS IN EDUCATION CONFERENCE, 2013. Proceedings... Oklahoma: IEEE, 2013.

HUOTARI, K.; HAMARI, J. Defining gamification: a service marketing perspective. In: INTERNATIONAL ACADEMIC MINDTREK CONFERENCE, 16., 2012, Tampere. Proceedings... Tampere: ACM, 2012. p. 17-22.

KACHNIEWSKA, M. Gamification in tourism: pitfalls and benefits. In: TOURISM IN THE AGE OF TRANSFORMATION, 2015. Proceedings... Varna: University of Economics, 2015.

KIRÁL'OVÁ, A. The place of gamification in tourism destination's marketing. In: INFORMATION TECHNOLOGY AND SOCIETY INTERACTION AND INTERDEPENDENCE, 23., 2015. Proceedings... Linz: Trauner Verlag, 2015. NEGRUŞA, A. L. et al. Exploring gamifica- 
tion techniques and applications for sustainable tourism. Sustainability, Basel, v. 7, n. 8, p. 11160-11189, 2015.

KOVACEVIC, I.; ZECEVIC, B.; VELJKOVIC, S. "Gamification” concept: theoretical framework and destination marketing management practice. Ekonomika Preduzeca, [s. 1.], v. 62, n. 5-6, p. 315-322, 2014. Disponível em: <http://doi.org/10.5937/ekopre1406315K>. Acesso em: 5 abr. 2017.

POPKIN, H. FarmVille invades the real world. NBC News, 6 jan. 2010. Disponível em: <http://nbcnews.to/2nbMr5f>. Acesso em: 16 abr. 2016.

SIGALA, M. Gamification for crowdsourcing marketing practices: applications and benefits in tourism. In: GARRIGOS-SIMON, F. J., GIL-PECHUÁN, I., ESTELLES-MIGUEL, S. (Eds.). Advances in Crowdsourcing. Cham: Springer, 2015a. p. 129-146. Disponível em: <http:// bit.ly/2oJrc7L>. Acesso em: 5 abr. 2017.

. The application and impact of gamification funware on trip planning and experiences: the case of TripAdvisor's funware. Electronic Markets, Leipzig, v. 25, n. 3, p. 189209, 2015b. Disponível em: <https://doi.org/10.1007/s12525-014-0179-1>. Acesso em: 5 abr. 2017.

USKOV, A.; SEKAR, B. Serious games, gamification and game engines to support framework activities in engineering: case studies, analysis, classifications and outcomes. In: IEEE INTERNATIONAL CONFERENCE ON ON ELECTRO/INFORMATION TECHNOLOGY (EIT), 2014. Proceedings... Milwaukee: IEEE, 2014 . p. 618-623.

$\mathrm{XU}, \mathrm{F}$. et al. Tourists as mobile gamers: gamification for tourism marketing. Journal of Travel \& Tourism Marketing, London, v. 33, n. 8, p. 1124-1142, 2015.

YAMAMOTO, R.; YOSHIN, T.; SONEHARA, N. Gamification effect of collection system for digital photographs with geographic information which utilizes land acquisition game. In: INTERNATIONAL CONFERENCE ON HUMAN-COMPUTER INTERACTION, 17., 2015. Proceedings... Los Angeles: Springer, 2015.

ZICHERMANN, G.; CUNNINGHAM, C. Gamification by design: implementing game mechanics in web and mobile apps. Newton: O’Reilly Media, 2010.

ZICHERMANN, G.; LINDER, J. The gamification revolution: how leaders leverage game mechanics to crush the competition. New York: Mc Graw-Hill Education, 2013.

Recebido em: 25/09/2016 Aprovado em: 25/02/2017 